\title{
Methanothermobacter - Biokatalysator für die Energiewende
}

SEIGO SHIMA, RUDOLF K. THAUER

MAX-PLANCK-INSTITUT FÜR TERRESTRISCHE MIKROBIOLOGIE, MARBURG

\section{Methanothermobacter is a thermophilic genus within the kingdom of}

Euryarchaeota. Chemolithoautotrophic growth on $\mathrm{H}_{2}$ and $\mathrm{CO}_{2}$ at $65^{\circ} \mathrm{C}$ is rapid and to high cell concentrations. Champions in this respect are the species $M$. thermautotrophicus and M. marburgensis, which were used to elucidate the unique biochemistry of methane formation from $\mathrm{H}_{2}$ and $\mathrm{CO}_{2}$. These two species are presently also being explored as biocatalysts in the industrial conversion of electrolytically produced $\mathrm{H}_{2}$ to "green" methane.

DOI: $10.1007 / \mathrm{s} 12268-021-1530-8$ (C) Die Autoren 2021

In einer Kläranlage in Urbana, Illinois, USA beginnt diese Geschichte. Aus dem anaeroben Schlamm isolierten Gregory Zeikus und Ralph Wolfe 1972 eine hitzeliebende Mikrobe, die in Abwesenheit von Sauerstoff aus Wasserstoff $\left(\mathrm{H}_{2}\right)$ und $\mathrm{CO}_{2}$ Methan $\left(\mathrm{CH}_{4}\right.$, Biogas oder Erdgas) bildet [1, 2]. Sie wächst bei $65^{\circ} \mathrm{C}$ optimal. Das Isolat wurde zunächst Methanobacterium thermoautotrophicum und später Methanothermobacter thermautotrophicus benannt, da es sich um eine wärmeliebende (thermophile), nur von $\mathrm{CO}_{2}$ als Kohlenstoffquelle lebende (autotrophe) Mikrobe handelt [1, 2], die wie ein Bakterium aussieht (Abb. 1, [3])

Die Entdeckung von $M$. thermautotrophicus 1972 war ein Meilenstein, weil sie erstmals zeigte, dass auch strikte Anaerobier bei Temperaturen von $65{ }^{\circ} \mathrm{C}$ optimal wachsen können; alle damals bekannten extrem Thermophilen - wie Bacillus acidocaldarius und die Gattungen Thermus, Thermoplasma und Sulfolobus - benötigen Sauerstoff zum Leben. Sie zeigte ferner, dass chemotrophe Mikroben auch ohne Sauerstoff autotroph leben können; alle damals beschriebenen Chemo-
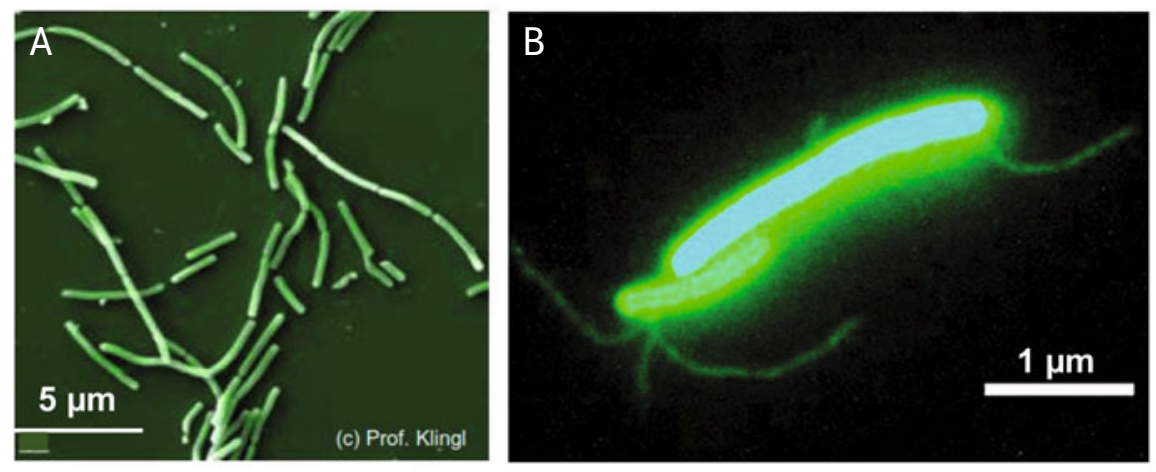

A Abb. 1: Methanothermobacter thermautotrophicus sind unbewegliche krumme Stäbchen. A, elektronenmikroskopische Aufnahme des patentierten $M$. thermautotrophicus-Stamms, den die Firma Electrochaea als Biokatalysator verwendet. (Foto (bearbeitet): Andreas Klingl, LMU München) B, fluoreszenzmikroskopische Aufnahme einer planktonischen M. thermautotrophicusZelle nach Anfärben mit AlexaFluor ${ }^{\circledR}-488$ [3]. Methanothermobacter heftet sich mit feinen Härchen (Fimbriae) an Oberflächen an. (mit freundlicher Genehmigung aus [3])

autotrophen, wie Knallgasbakterien und nitrifizierende Bakterien, benötigen Sauerstoff.

Heute gibt es acht beschriebene Methanothermobacter-Spezies. Sie wurden aus anaeroben Klärschlämmen, industriellen thermophilen Biogasanlagen, heißen Quellen oder Quellwasser von Gasfeldern isoliert und wachsen alle auf $\mathrm{H}_{2}$ und $\mathrm{CO}_{2}$ mit einem Temperaturoptimum um $65^{\circ} \mathrm{C}$. Ihre Genome sind

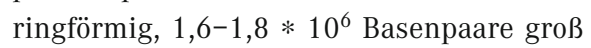
und haben einem DNA-G+C-Gehalt um die 50 Prozent. Wichtigste Spezies sind $M$. thermautotrophicus und $M$. marburgensis (M. marburgensis wurde zunächst Methanobacterium thermoautotrophicum, Stamm Marburg, genannt [2]).

Beide Spezies vermehren sich exponentiell mit Verdoppelungszeiten von rund zwei Stunden zu höheren Zelldichten als alle anderen bisher bekannten Methanbildner. Deshalb gelten sie als Modellorganismen für Untersuchungen der Biochemie der Methanogenese aus $\mathrm{H}_{2}$ und $\mathrm{CO}_{2}$ [4, 5]. Sie dienen als Biokatalysatoren in Pilotanlagen zur Umsetzung von $\mathrm{H}_{2}$ und $\mathrm{CO}_{2} \mathrm{zu}$ Methan für energetische Zwecke.

\section{Methanothermobacter sind Archaea}

Ende der 1970er-Jahre erkannte Karl Woese, dass es zwei Arten von Prokaryoten gibt: Bacteria und Archaea, die nur sehr entfernt miteinander verwandt sind. Methanothermobacter und alle anderen anaeroben Methanbildner gehören zu den Archaea. Dazu passt der frühe Befund, dass $M$. thermautotrophicus eine Gram-positive Zellwand ohne den Wandbaustoff Murein hat und deshalb nicht durch Penicillin im Wachstum gehemmt wird. Anstatt aus Murein besteht die Zellwand von M. thermautotrophicus aus einem mehrschichtigen Pseudomurein-Sacculus. Auch die Beschaffenheit der Cytoplasmamembran (aus Isoprenoidlipiden) und der Aufbau der RNA-Polymerase deuteten früh darauf hin, dass $M$. thermautotrophicus kein Bakterium, sondern ein Archaeon ist.

Die Gattung Methanothermobacter gehört zur Familie der Methanobacteriaceae, die wiederum zu der Ordnung der Methanobacte- 
riales gehört, eine von inzwischen acht methanogenen Ordnungen im Reich der Euryarchaeota.

\section{Energie und Kohlenstoff nur aus $\mathrm{H}_{2}$ und $\mathrm{CO}_{2}$}

Die farblosen unbeweglichen Archaea leben optimal bei etwa $65^{\circ} \mathrm{C}$, pH 7 und niedrigem Salzgehalt auf $\mathrm{H}_{2}$ und $\mathrm{CO}_{2}$ als einzigen Energie- und Kohlenstoffquellen. Die meisten Spezies wachsen in rein mineralischem Medium ohne Zusätze von Vitaminen, Aminosäuren oder Hefeextrakt. Methanothermobacter sind bezüglich ihres Energiestoffwechsels lithotroph (anorganischer Elektronendonator) und bezüglich ihres Baustoffwechsels autotroph (Zellkohlenstoff aus $\mathrm{CO}_{2}$ ).

Die Cytoplasmamembran von Methanothermobacter ist für $\mathrm{H}_{2}, \mathrm{CO}_{2}$ und Methan durchlässig, nicht aber für Zucker, Aminosäuren, Pyruvat und Acetat, für die es auch keine Transportsysteme gibt. Es fehlen Enzyme, die diese Verbindungen in der Zelle verstoffwechseln könnten. Infolgedessen gibt es für Methanothermobacter keine Alternativen zur Methanbildung aus $\mathrm{H}_{2}$ und $\mathrm{CO}_{2}$ als Energiequelle und zur autotrophen $\mathrm{CO}_{2}$-Fixierung. Methanothermobacter sind daher obligat lithoautotroph, allerdings mit einer Einschränkung: Einige Stämme können nämlich Ameisensäure (Formiat) anstelle von $\mathrm{H}_{2}$ und $\mathrm{CO}_{2}$ zum Wachstum verwenden, wobei allerdings die Reduktion der Ameisensäure zu Methan über $\mathrm{CO}_{2}$ läuft.

Als Stickstoffquelle verwendet die Mikrobe ausschließlich $\mathrm{NH}_{3}$ und als Schwefelquelle ausschließlich $\mathrm{H}_{2} \mathrm{~S}$. Als Spurenelemente benötigt sie Eisen, Kobalt, Nickel, Molybdän und/oder Wolfram, nicht aber Mangan, Kupfer und Selen [6]. Wie alle Lebewesen brauchen Methanothermobacter auch Kalium- und Magnesiumionen zum Wachstum. Methanbildung und Wachstum dieser Archaea sind von Natriumionen abhängig, obwohl Methanothermobacter keine marinen Organismen sind.

\section{Natriumionen für den Stoffwechse}

Der Energie- und Baustoffwechsel von Methanothermobacter sind in Abbildung 2 skizziert $[5,7]$. Am Stoffwechsel sind sechs Coenzyme beteiligt, die erstmals in Methanothermobacter entdeckt wurden. Es handelt sich dabei um die $\mathrm{C}_{1}$-tragenden Coenzyme Methanofuran (MFR), Tetrahydromethanopterin $\left(\mathrm{H}_{4} \mathrm{MPT}\right)$ und Coenzym M (HS-CoM) und die Elektronenüberträger Polyferredoxin $(\mathrm{pFd})$, Coenzym $\mathrm{F}_{420}\left(\mathrm{~F}_{420}\right)$ und Coenzym B (HS$\mathrm{CoB}$ ). Die Reduktion von $\mathrm{CO}_{2}$ (Kohlenstoff-

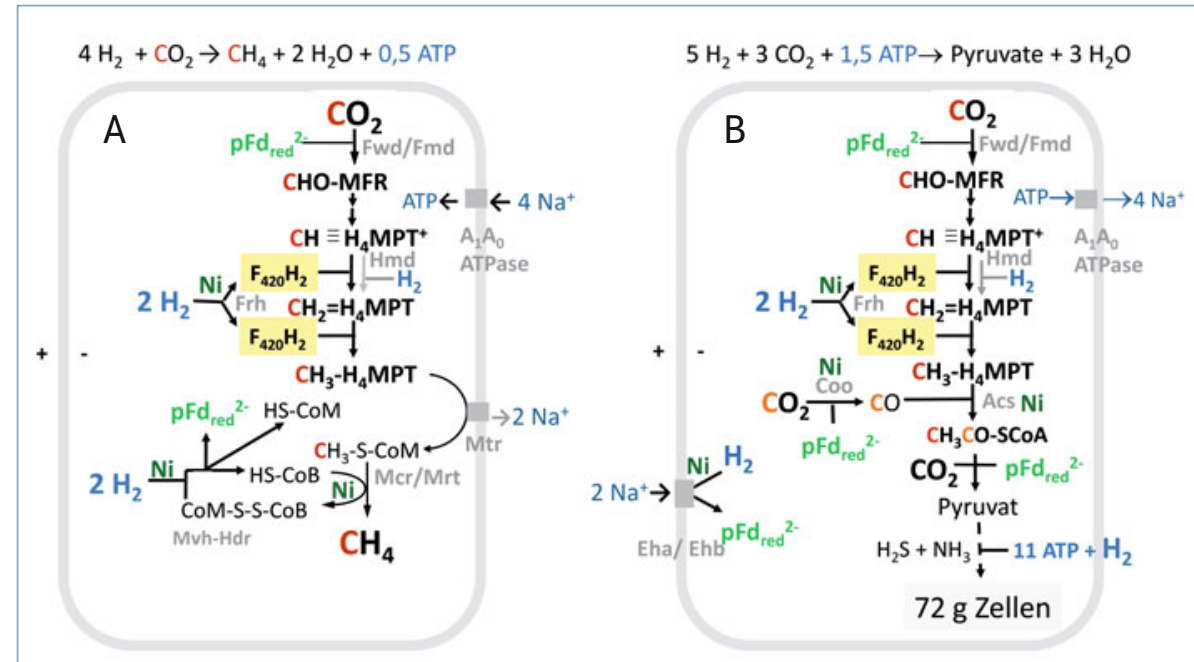

$\triangle$ Abb. 2: Schematische Darstellung des Energiestoffwechsels (A) und des autotrophen Baustoffwechsels (B) von Methanothermobacter bei Wachstum auf $\mathrm{H}_{2}$ und $\mathrm{CO}_{2}$. In der Berechnung der benötigten Menge ATP für die Synthese von $72 \mathrm{~g}$ Zellen aus $3 \mathrm{CO}_{2}$ wurde angenommen, dass die Zellen zu $50 \%$ aus Kohlenstoff bestehen und dass der theoretische ATP-Wachstumsertrag $5 \mathrm{~g}$ Zellen/mol ATP ist. Der Wachstumsertrag pro mol Methan wurde für $M$. marburgensis zu 2,5 g Zellen/mol Methan bestimmt [5]. MFR: Methanofuran; $\mathrm{H}_{4}$ MPT: Tetrahydromethanopterin; HS-CoM: Coenzym M; HS-CoB: Coenzym B; $\mathrm{F}_{420}$ : Coenzym $\mathrm{F}_{420}$; pFd: Polyferredoxin, das pro [4Fe4S]-Cluster jeweils ein Elektron überträgt; Acs: Acetyl-CoA-Synthase/Decarbonylase; ATPase: ATP-Synthetase; Coo: Kohlenmonoxid-Dehydrogenase; Eha und Ehb: energiekonservierende [NiFe]Hydrogenasen; Frh: $\mathrm{F}_{420}$-reduzierende [NiFe]-Hydrogenase; Fwd und Fmd: Wolfram- bzw. Molybdän-abhängige Formylmethanofuran-Dehydrogenase; Hmd: [Fe]-Hydrogenase; Mcr und Mrt: Methyl-Coenzym M-Reduktase; Mtr: Methyltransferase; Mvh-Hdr: Methylviologen-reduzierender [NiFe]-Hydrogenase-Heterodisulfid-Reduktase-Komplex.

Oxidationsstufe +4$)$ zu Methan (-4) erfolgt über Formyl-MFR (+2), Formyl-H ${ }_{4}$ MPT (+2), Methenyl- $\mathrm{H}_{4}$ MPT (+2), Methylen- $\mathrm{H}_{4}$ MPT (0), Methyl- $\mathrm{H}_{4}$ MPT (-2) und Methyl-Coenzym M $(-2)$ als Zwischenprodukte. Reduziertes Polyferredoxin $\left(\mathrm{pFd}_{\text {red }}{ }^{2-}\right), \mathrm{F}_{420} \mathrm{H}_{2}$ und HS-CoB dienen dabei als Elektronendonatoren, die ihrerseits durch $\mathrm{H}_{2}$ re-reduziert werden.

Die bei der Reduktion von $\mathrm{CO}_{2} \mathrm{zu}$ Methan freiwerdende Energie wird zum Aufbau eines elektrochemischen $\mathrm{Na}^{+}$-Potenzials verwendet, das seinerseits die endergone Synthese von ATP (Abb. 2A) und die endergone Reduktion von Polyferredoxin ( $\mathrm{pFd}$ ) mit $\mathrm{H}_{2}$ treibt (Abb. 2B, [7]).

Im Falle des Baustoffwechsels wird $\mathrm{CO}_{2} \mathrm{zu}$ Kohlenmonoxid (CO) (+2) reduziert, das mit Methyl- $\mathrm{H}_{4}$ MPT (-2) (aus dem Energiestoffwechsel) und Coenzym A zu Acetyl-CoA reagiert, das wiederum reduktiv zu Pyruvat carboxyliert wird. Von Pyruvat aus werden ATP-abhängig fast alle Zellbausteine synthetisiert (Abb. 2B).

Der $\mathrm{Na}^{+}$-abhängige Energiestoffwechsel von $\mathrm{CO}_{2}$ und $\mathrm{H}_{2}$ zu Methan, wie er in Abbildung 2A aufgezeichnet ist, findet sich mit kleineren Modifikationen in den hydrogenophilen Mitgliedern aller Ordnungen von methanogenen Archaea wieder. Nur in der Ordnung Metha- nosarcinales ist der Kopplungsmechanismus wesentlich anders. Während im Energiestoffwechsel von Methanothermobacter die endergone Reduktion von Polyferredoxin $(\mathrm{pFd}) \mathrm{mit}$ $\mathrm{H}_{2}$ an die exergone Reduktion von CoM-S-S$\mathrm{CoB}$ mit $\mathrm{H}_{2}$ durch Flavin-basierte Elektronenbifurkation gekoppelt ist (Abb. 2A, [8]), erfolgt diese Kopplung in Methanosarcina-Spezies chemiosmotisch [5].

\section{Der Nickelbedarf war eine Zufalls- entdeckung}

Bei Wachstumsversuchen mit M. marburgensis beobachteten Peter Schönheit et al. 1979 zufällig, dass Kulturen in Rührfermentern mit Anteilen aus Edelstahl besser wachsen als solche in Fermentern ohne Edelstahlanteile [6]. Analysen des Edelstahls ergab eine Zusammensetzung aus Eisen, Nickel und Chrom. Es stellte sich schnell heraus, dass Nickel das Wachstum beschleunigte, was eine große Überraschung war. Damals glaubte man noch, dass Nickel keine biologische Funktion in Mikroorganismen hat. Dass Methanothermobacter-Spezies zuvor überhaupt wuchsen, lag wohl an Spuren von Nickel in den verwendeten Medien.

Die Nickelabhängigkeit des Wachstums von Methanothermobacter liegt an den min- 
destens acht Nickelenzymen, die am Energie(Abb. 2A) und Baustoffwechsel (Abb. 2B) beteiligt sind: vier unterschiedliche [NiFe]Hydrogenasen (Frh, Mvh, Eha und Ehb), zwei Methyl-Coenzym-M-Reduktasen (Mcr oder Mrt), Kohlenmonoxid-Dehydrogenase (Coo) und Acetyl-CoA-Synthase/Decarbonylase (Acs) (Abb. 2). Alle methanogenen Archaea verfügen zumindest über eine Methyl-Coenzym-M-Reduktase, weshalb das Wachstum aller methanogenen Archaea nickelabhängig ist.

Inzwischen wissen wir, dass die meisten Bakterien und Archaeen Nickel zum Wachstum benötigen, allerdings in sehr viel geringeren Mengen als Methanothermobacter.

\section{Methanothermobacter vertragen keinen Sauerstoff}

Schon kleinste Spuren von $\mathrm{O}_{2}$ hemmen Methanothermobacter im Wachstum. Das Vereinzeln von Methanothermobacter auf Agar-Oberflächen ist nur möglich, wenn die Gasphase über dem Agar viel weniger als 10 ppm $\mathrm{O}_{2}$ enthält. Methanothermobacter enthalten keine Häm-Proteine und damit auch keine Katalase. Sie enthalten aber eisenabhängige Superoxid-Dismutase (SOD), um sich vor dem Hyperoxid-Anion-Radikal zu schützen, und $\mathrm{F}_{420} \mathrm{H}_{2}$-Oxidase (FprA), die die VierElektronen-Reduktion von $\mathrm{O}_{2} \mathrm{zu} \mathrm{H}_{2} \mathrm{O}$ katalysiert [9].

Die Zellen von Methanothermobacter sind voll von Eisen-Schwefel-Proteinen, die im reduzierten Zustand spontan mit $\mathrm{O}_{2}$ reagieren. Dazu gehören Polyferredoxine $(\mathrm{pFd})$ [10], Formyl-Methanofuran-Dehydrogenasen (Fwd und Fmd) [10], Heterodisulfid-Reduktase (Hdr) [11] und [NiFe]-Hydrogenasen (Frh, Mvh, Eha und Ehb) [12], die an der Reduktion von $\mathrm{CO}_{2}$ mit $\mathrm{H}_{2}$ zu Methan (Abb. 2A) und an der autotrophen $\mathrm{CO}_{2}$-Fixierung (Abb. 2B) beteiligt sind. Im Falle von reduzierten Polyferredoxinen, deren Eisen-Schwefel-Cluster exponiert sind, reagiert $\mathrm{O}_{2}$ direkt mit einem der reduzierten Eisen-Schwefel-Cluster, die nur ein Elektron auf einmal übertragen. $\mathrm{O}_{2}$ wird dabei daher zunächst zum HyperoxidAnion-Radikal $\left(\mathrm{O}_{2}^{-}\right)$reduziert, das dann entweder weiter $\mathrm{zu} \mathrm{H}_{2} \mathrm{O}_{2}$ reduziert wird oder beschleunigt durch $\mathrm{SOD}$ zu $\mathrm{O}_{2}$ und $\mathrm{H}_{2} \mathrm{O}_{2}$ zerfällt. Diese „reaktiven Sauerstoffspezies“ sind dann für die Zellen toxisch. Im Falle der anderen Eisen-Schwefel-Proteine, die weniger exponierte Eisen-Schwefel-Cluster haben, dürfte der Hauptangriffspunkt von $\mathrm{O}_{2}$ das reduzierte Übergangsmetall (Mo, W, oder Ni) oder das reduzierte Flavin im aktiven Zentrum sein.

Es gibt in Methanothermobacter aber auch Enzyme ohne Eisen-Schwefel-Cluster, die mit $\mathrm{O}_{2}$ spontan reagieren, so Methyl-Coenzym-MReduktase (Mcr und Mrt), die die methanbildende Reaktion im Energiestoffwechsel katalysiert (Abb. 2A). Ni(I)F $\mathrm{F}_{430}$ (ein Nickel-Tetrapyrrol) im aktiven Zentrum dieses Schlüsselenzyms wird aufgrund seines negativen Redoxpotenzials und seiner leichten Zugänglichkeit im Protein durch Spuren von $\mathrm{O}_{2}$ oxidiert, wodurch das Enzym inaktiviert wird [13].

\section{Methanbildungsraten in Bioreaktoren}

Bei ausreichender Begasung verdoppeln sich M. marburgensis-Zellen in Rührfermentern bei $65^{\circ} \mathrm{C}$ in 1,6 Stunden bis zu einer Zellkonzentration von etwa 2,5 Gramm (Trockengewicht) pro Liter Kultur. Pro Mol gebildeten Methan werden etwa 1,6 Gramm Zellen gebildet. Bei höheren Zellkonzentrationen limitiert die Massentransferrate von $\mathrm{H}_{2}$ aus der Gas- in die Flüssigphase das Wachstum. Unter diesen energielimitierenden Bedingungen steigt der Wachstumsertrag auf etwa 2,5 Gramm Zellen pro Mol gebildeten Methan an. In Bioreaktoren mit höheren Gastransferraten verschiebt sich die Energielimitierung zu höheren Zelldichten [5].

Bei einer Zellkonzentration von 2,5 Gramm pro Liter Kultur produzieren die Zellen in den verwendeten Rührfermentern Methan mit einer Rate um 10 mMol Methan bzw. 0,224 Liter Methan pro Liter Kultur und
Minute. Diese Rate lässt sich in Bioreaktoren mit höheren Gastransferraten noch um einen Faktor von 2-3 steigern. Damit kommen die Raten in einen Bereich, der für die Umsetzung von $\mathrm{H}_{2}$ und $\mathrm{CO}_{2}$ zu Methan für energetische Zwecke interessant ist.

Es gibt wenige methanogene Archaea wie Methanothermococcus thermolithotrophicus $\left(65{ }^{\circ} \mathrm{C}\right)(\mathrm{td}=30 \mathrm{~min})$ [11], die unter vergleichbaren Bedingungen im Rührfermenter schneller als Methanothermobacter wachsen, dies aber nur zu wesentlich geringeren Zellkonzentrationen tun. Zudem ist M. thermolithotrophicus ein mariner Mikroorganismus, der höhere Konzentrationen von $\mathrm{NaCl}$ (4 \%) zum Wachsen braucht, was im Bioreaktor aus Edelstahl die Korrosion beschleunigt.

\section{Power-to-Gas mit Methanothermo- bacter}

Aufgrund ihrer hohen metabolischen Aktivität und Fähigkeiten, bis zu hohen Zellkonzentration zu wachsen, werden $M$. thermautotrophicus und $M$. marburgensis seit einiger Zeit darauf hin untersucht, ob sie als Biokatalysatoren bei der Umwandlung von elektrolytisch gewonnenem Wasserstoff mit $\mathrm{CO}_{2}$ zu Methan im industriellen Maßstab eingesetzt werden können. Methan lässt sich leichter speichern als $\mathrm{H}_{2}$. Und bei der Verbrennung von Methan wird pro Mol 3,33-mal mehr Energie frei als bei der Verbrennung von $\mathrm{H}_{2}$. Die Münchener Startup-Firma Electrochaea GmbH (www.electrochaea.com) hat dieses Power-to-Gas-Verfahren zur Produktion von „grünem“ Methan erfolgreich

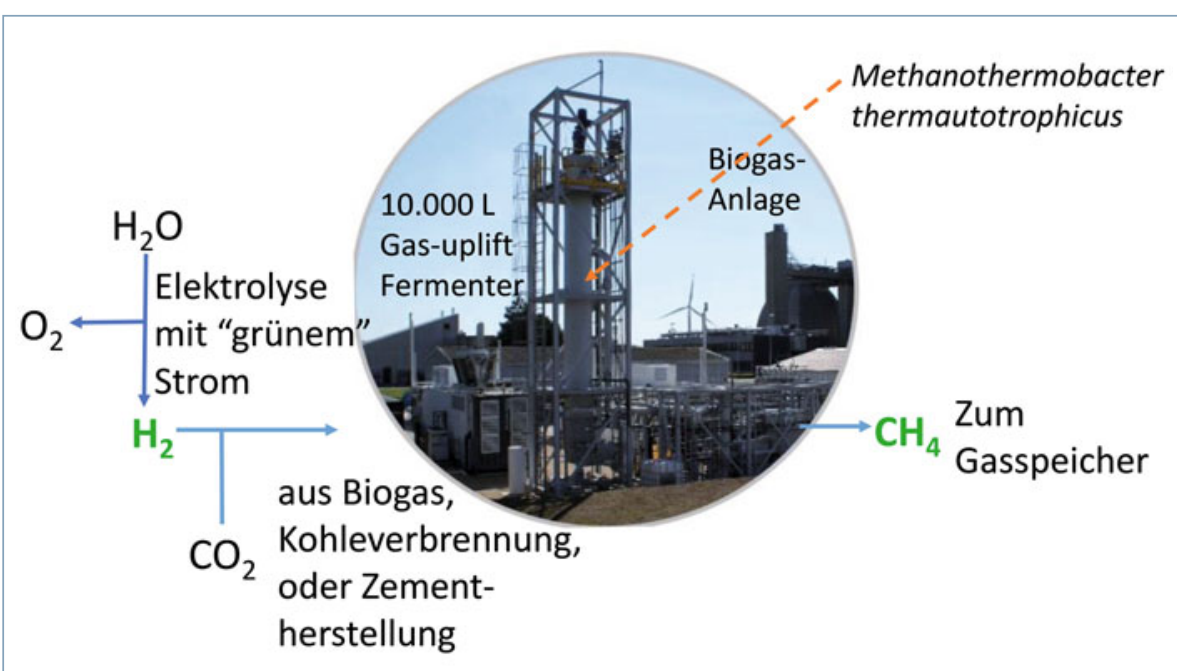

A Abb. 3: Power-to-Gas: 10.000-Liter-Fermenter-Pilotanlage nahe Kopenhagen, Dänemark, der Firma Electrochaea mit einem patentierten Methanothermobacter thermautotrophicus-Stamm als Biokatalysator. Die Anlage mit einem Füllvolumen von 3.600 Liter produziert 50 Kubikmeter Methan pro Stunde (www.electrochaea.com) - energetisch etwas mehr als 40 Liter Benzin/Diesel pro Stunde. 
entwickelt (Abb. 3): Mit Strom aus erneuerbaren Energien wird Wasser $\mathrm{zu} \mathrm{H}_{2}$ und $\mathrm{O}_{2}$ elektrolysiert und das so gewonnene $\mathrm{H}_{2}$ mit $\mathrm{CO}_{2} \mathrm{zu}$ Methan umgesetzt, wobei das benötigte $\mathrm{CO}_{2}$ aus jeder beliebigen Quelle, z. B. aus Biogasanlagen, Kohlekraftwerken oder der Zementindustrie stammen kann. Als Katalysator dient ein Stamm von $M$. thermautotrophicus, der unter Katalyse-Bedingungen den Energie- vom Baustoffwechsel weitgehend entkoppeln kann, damit fast alles $\mathrm{CO}_{2}$ $\mathrm{zu}$ Methan reduziert wird und nur wenig Zellmasse entsteht. Auch die Krajete $\mathrm{GmbH}$ in Österreich (www.krajete.com) setzt mit einem stärker technischen Ansatz M. marburgensis erfolgreich für das gleiche Ziel ein. Leider gibt es für Methanothermobacter noch kein genetisches System, um gezielt Stämme zu konstruieren, die als Biokatalysator geeigneter sind als der Wildtyp.

\section{Literatur}

[1] Zeikus JG, Wolfe RS (1972) Methanobacterium thermoautotrophicus sp. nov., an anaerobic, autotrophic, extreme thermophile. J Bacteriol 109: 707-713

[2] Wasserfallen A, Nölling J, Pfister P et al. (2000) Phylogenetic analysis of 18 thermophilic Methanobacterium isolates supports the proposals to create a new genus,

Methanothermobacter gen. nov., and to reclassify several isolates in three species, Methanothermobacter thermautotrophicus comb. nov., Methanothermobacter wolfeii comb. nov., and Methanothermobacter marburgensis sp nov. Int J Syst Evol Micr 50: 43-53

[3] Thoma C, Frank M, Rachel R et al. (2008) The Mth60 fimbriae of Methanothermobacter thermoautotrophicus are functional adhesins. Environ Microbiol 10: 2785-2795

[4] Wolfe RS (1993) An historical overview of methanogenesis. In: Ferry JG (Hrsg.) Methanogenesis: Ecology, Phzsiology, Biochemistry and Genetics. Chapman and Hall, New York, London, 1-32
[5] Thauer RK, Kaster AK, Seedorf H et al. (2008) Methanogenic archaea: ecologically relevant differences in energy conservation. Nat Rev Microbiol 6: 579-591 [6] Schönheit P, Moll J, Thauer RK (1979) Nickel, cobalt, and molybdenum requirement for growth of Methanobacterium thermoautotrophicum. Arch Microbiol 123: 10--107

[7] Gottschalk G, Thauer RK (2001) The $\mathrm{Na}^{+}$-translocating methyltransferase complex from methanogenic archaea. Biochim Biophys Acta 1505: 28-36

[8] Buckel W, Thauer RK (2018) Flavin-based electron bifurcation, ferredoxin, flavodoxin, and anaerobic respiration with protons (Ech) or $\mathrm{NAD}^{+}$(Rnf) as electron acceptors: A historical review. Front Microbiol 9: 401

[9] Engilberge S, Wagner T, Carpentier P et al. (2020) Krypton-derivatization highlights 02 -channeling in a fourelectron reducing oxidase. Chem Commun 56: 10863-10866 [10] Wagner T, Ermler U, Shima S (2016) The methanogenic $\mathrm{CO}_{2}$ reducing-and-fixing enzyme is bifunctional and contains 46 [4Fe-4S] clusters. Science 354: 114-117

[11] Wagner T, Koch J, Ermler U et al. (2017) Methanogenic heterodisulfide reductase (HdrABC-MvhAGD) uses two noncubane [4Fe-4S] clusters for reduction. Science 357: 699-702 [12] Thauer RK, Kaster AK, Goenrich M et al. (2010) Hydrogenases from methanogenic archaea, Nickel, a novel cofactor, and $\mathrm{H}_{2}$-storage. Annu Rev Biochemistry 79: 507-536
[13] Thauer RK (2019) Methyl (Alkyl)-coenzyme M reductases: Nickel $\mathrm{F}_{430}$-containing enzymes involved in anaerobic methane formation and in anaerobic oxidation of methane or of short chain alkanes. Biochemistry 58: 5198-5220

Funding note: Open Access funding enabled and organized by Projekt DEAL. Open Access: Dieser Artikel wird unter der Creative Commons Namensnennun 4.0 International Lizenz veröffentlicht, welche die Nutzung, Vervielfältigung, Bearbeitung, Verbreitung und Wiedergabe in jeglichem Medium und Format erlaubt, sofern Sie den/die ursprünglichen Autor(en) und die Quelle ordnungsgemäß nennen, einen Link zur Creative Commons Lizenz beifügen und angeben, ob Änderungen vorgenommen wurden. Die in diesem Artikel enthaltenen Bilder und sonstiges Drittmaterial unterliegen ebenfalls der genannten Creative Commons Lizenz, sofern sich aus der Abbildungslegende genannten Creative nach gesetzlichen Vorschriften erlaubt ist, ist für die oben aufgeführten nich Weiterverwendungen des Materials die Einwilligung des jeweiligen

Rechteinhabers einzuholen. Weitere Details zur Lizenz entnehmen Sie bitte der Lizenzinformation auf http://creativecommons.org/licenses/by/4.0/deed.de.

\section{Korrespondenzadresse:}

Prof. Dr. Rudolf K. Thauer

Max-Planck-Institut für terrestrische Mikrobiologie Karl-von-Frisch-Straße 10

D-35043 Marburg

thauer@mpi-marburg.mpg.de

Seigo Shima
1980-1983 Studium der Landwirtschaft. 1991 PhD (Agriculture) University of Tokyo,
Japan. 1985-1994 Wissenschaftler bei CRIEPI, Japan. 1994-1995 Humboldt Stipen-
diat am Max-Planck-Institut für terrestrische Mikrobiologie in Marburg bei Prof. Dr. R.
Thauer. Dort 1995-2014 Arbeitsgruppenleiter und seit 2014 Leiter der unabhängigen
Arbeitsgruppe Microbial Protein Structure. Seit 2012 Gastprofessor an der Hokkaido
University in Japan.

